\title{
Sex differences in outcomes with transcatheter aortic valve replacement
}

\author{
Feng Qian, Edward L. Hannan
}

Department of Health Policy, Management \& Behavior, School of Public Health, State University of New York, University at Albany, Albany, NY, USA Correspondence to: Feng Qian, MD, PhD. Department of Health Policy, Management \& Behavior, School of Public Health, University at AlbanyState University of New York, GEC Room 169, One University Place, Rensselaer, NY 12144, USA. Email: fqian@albany.edu.

Provenance: This is a Guest Editorial commissioned by Section Editor Busheng Zhang, MD, PhD (Department of Cardiac Surgery, Shanghai Chest Hospital, Shanghai Jiaotong University, Shanghai, China).

Comment on: Chandrasekhar J, Dangas G, Yu J, et al. Sex-Based Differences in Outcomes With Transcatheter Aortic Valve Therapy: TVT Registry From 2011 to 2014. J Am Coll Cardiol 2016;68:2733-44.

Submitted Mar 25, 2017. Accepted for publication Apr 01, 2017.

doi: 10.21037/atm.2017.04.09

View this article at: http://dx.doi.org/10.21037/atm.2017.04.09

Transcatheter aortic valve replacement (TAVR) has emerged as a new standard of care for treating patients with symptomatic aortic stenosis (AS) and inoperable status. It was approved by the US Food and Drug Administration (FDA) in November 2011 (1). Ten months later, TAVR was approved by the FDA for treatment of high-risk but operable status patients with AS. Since then, TAVR has rapidly been adopted in clinical practice for the treatment of symptomatic AS patients at high risk for surgery. However, it remains unclear and unresolved whether there are sex-related differences in outcomes for patients who undergo TAVR.

To address this question, Chandrasekhar et al. used 4-year data (from 2011 through 2014) from the Society of Thoracic Surgeons/American College of Cardiology Transcatheter Valve Therapy (STS/ACC TVT) registry, the national mandatory TAVR registry in the US, to study both in-hospital and 1-year TAVR outcomes in male and female patients (2). This paper included 11,808 female (49.9\%) and 11,844 male (51.1\%) patients who underwent TAVR and thus represented the largest observational study that examined sex-specific differences at presentation, in-hospital and after 1 year. The authors showed that compared with males, female patients undergoing TAVR tended to have fewer comorbidities but a different risk profile such as older age, more frailty, higher STS score, more moderate or severe mitral valve regurgitation and a higher rate of porcelain aorta. These differences at baseline were consistent with findings from prior studies (3-6). Such differences suggest a strong need to apply patient-centered TAVR risk/benefit assessment metrics for female and male patients. For procedural characteristics and outcomes, this study reported that female TAVR patients were more likely to undergo non-transfemoral approach relative to males (45.0\% vs. $34.0 \%$ ), which was different from a recent large scale meta-analysis study (5). One explanation could be that the meta-analysis paper included five previous studies using a total of 11,310 patients from multiple countries' TAVR registries (7-11) whereas Chandrasekhar's paper only used the STS/ACC TVT registry in the US. More importantly, Chandrasekhar et al. reported for the first time that female patients experienced a higher rate of coronary obstruction and conversion to open heart surgery (2). This highlights the importance of adjusting for the sexrelated anatomical differences such as smaller annuli and left ventricular outflow tract dimensions in comparing outcomes of female and male patients undergoing TAVR procedure to help determine if these differences are the reason why women have higher rates of obstruction and conversion. For in-hospital outcomes, Chandrasekhar et al. found that compared with male patients, females had a significantly increased rate of vascular complications [4.39\% vs. 8.27\%; adjusted hazard ratio (AHR), 1.70; 95\% CI, 1.34-2.14; $\mathrm{P}<0.001]$ and a trend for higher bleeding rate (5.96\% vs. 8.01\%; AHR, 1.19; 95\% CI, 0.99-1.44; $\mathrm{P}=0.06$ ). These observed sex-specific differences in perioperative 
outcomes are in line with preexisting literature (3-6). But, Chandrasekhar et al. also demonstrated that female patients had much lower 1-year all-cause mortality rate relative to males (21.3\% vs. 24.5\%; AHR, 0.73; 95\% CI, 0.63-0.85; $\mathrm{P}<0.001)$. This finding is consistent with the vast majority of previous studies $(3,5-7,12)$ although there are very few prior studies showing similar or even higher mortality in female TAVR patients relative to males $(8,13)$.

There are some possible explanations for why women were found to have superior long-term TAVR outcomes that may be related to the methodology of the study by Chandrasekhar et al. First, at any fixed age, women have a longer life expectancy than men. For example, the average life expectancy of 82 years old (the mean age of TAVR patients in the study) women is 8.5 years compared with 7.3 years for men (14). Although it would probably not alter the significant differences found in the study, this survival difference should ideally be accounted for in the analyses.

Second, as noted above, there is a large difference in the use of transfemoral access in the study, with a utilization of $65 \%$ for men and $55 \%$ for women. Since transfemoral access is generally associated with superior outcomes (15), this would appear to convey an added survival advantage to men in the analyses. However, access site is controlled for in the risk-adjustment process, so this advantage is removed. There are a few reasons why women may have had lower use of transfemoral access, including available sheath sizes and center preference, as mentioned by the authors. Another possible reason is more calcification of femoral arteries combined with smaller vessels, and it is arguable as to whether this should be controlled for when examining survival differences between the sexes. It would be interesting to compare survival differences separately for transfemoral and non-transfemoral access sites.

It is also notable that although women had higher 2-year survival rates, they had significantly higher rates of inhospital coronary destruction/compression, unplanned other cardiac surgery, and major vascular complications, as well as higher unadjusted rates of in-hospital death, myocardial infarction (MI) and stroke. For example, the survival curves in the central illustration show that crude mortality and MI rates for women are higher until about 4 months after TAVR. This emphasizes the need to study the causes of mortality, as the authors recommend. It would be interesting to identify whether more late deaths among men occur because of TAVR complications or failure, or whether it was primarily due to a cause unrelated to TAVR.
One possible reason why the survival curves separated over time in favor of women may be related to differences in medication adherence. For example, although the evidence on sex-related medication adherence is mixed, Manteuffel et al. found that for patients with diabetes and select cardiovascular conditions, women were significantly more likely than men to use one or more medications during the analysis period $(68 \%$ vs. $59 \%, \mathrm{P}<0.001)$, although men were more likely to receive the medication treatment and monitoring recommended by clinical guidelines (16). It should be noted that other studies have found either differences in favor of higher adherence for men (17) or no difference in adherence (18) by sex.

It is also important that the conclusion that women have better longer-term outcomes than men is very dependent on the finding that women are sicker than men at the time of procedure being performed, and that women fare better in the long run despite having worse (or at least not better) short-term outcomes. As the authors note, women were sicker in some respects (higher rate of porcelain aorta, lower glomerular filtration rate, higher STS score) but were less sick in other respects (lower prevalence of coronary artery disease, atrial fibrillation and diabetes). The statistical model used by the authors was different than earlier models using the STS score (19) or the New York statistical model for surgical valve patients (20), and it would be interesting to see if the use of those two statistical models would have yielded similar results.

In conclusion, Chandrasekhar et al. are to be congratulated for shedding more light on TAVR outcome differences between the sexes, and their study raises some interesting questions to be addressed in future studies.

\section{Acknowledgements}

None.

\section{Footnote}

Conflicts of Interest: The authors have no conflicts of interest to declare.

\section{References}

1. Mack MJ, Brennan JM, Brindis R, et al. Outcomes following transcatheter aortic valve replacement in the United States. JAMA 2013;310:2069-77.

2. Chandrasekhar J, Dangas G, Yu J, et al. Sex-Based 
Differences in Outcomes With Transcatheter Aortic Valve Therapy: TVT Registry From 2011 to 2014. J Am Coll Cardiol 2016;68:2733-44.

3. Hayashida K, Morice MC, Chevalier B, et al. Sex-related differences in clinical presentation and outcome of transcatheter aortic valve implantation for severe aortic stenosis. J Am Coll Cardiol 2012;59:566-71.

4. Williams M, Kodali SK, Hahn RT, et al. Sex-related differences in outcomes after transcatheter or surgical aortic valve replacement in patients with severe aortic stenosis: Insights from the PARTNER Trial (Placement of Aortic Transcatheter Valve). J Am Coll Cardiol 2014;63:1522-8.

5. O'Connor SA, Morice MC, Gilard M, et al. Revisiting Sex Equality With Transcatheter Aortic Valve Replacement Outcomes: A Collaborative, Patient-Level Meta-Analysis of 11,310 Patients. J Am Coll Cardiol 2015;66:221-8.

6. Kodali S, Williams MR, Doshi D, et al. Sex-Specific Differences at Presentation and Outcomes Among Patients Undergoing Transcatheter Aortic Valve Replacement: A Cohort Study. Ann Intern Med 2016;164:377-84.

7. Humphries KH, Toggweiler S, Rodés-Cabau J, et al. Sex differences in mortality after transcatheter aortic valve replacement for severe aortic stenosis. J Am Coll Cardiol 2012;60:882-6.

8. Al-Lamee R, Broyd C, Parker J, et al. Influence of gender on clinical outcomes following transcatheter aortic valve implantation from the UK transcatheter aortic valve implantation registry and the National Institute for Cardiovascular Outcomes Research. Am J Cardiol 2014;113:522-8.

9. Gilard M, Eltchaninoff H, Iung B, et al. Registry of transcatheter aortic-valve implantation in high-risk patients. N Engl J Med 2012;366:1705-15.

10. Tamburino C, Capodanno D, Ramondo A, et al. Incidence and predictors of early and late mortality after transcatheter aortic valve implantation in 663 patients with

Cite this article as: Qian F, Hannan EL. Sex differences in outcomes with transcatheter aortic valve replacement. Ann Transl Med 2017;5(16):330. doi: 10.21037/atm.2017.04.09 severe aortic stenosis. Circulation 2011;123:299-308.

11. Kodali SK, Williams MR, Smith CR, et al. Two-year outcomes after transcatheter or surgical aortic-valve replacement. N Engl J Med 2012;366:1686-95.

12. Zahn R, Gerckens U, Linke A, et al. Predictors of oneyear mortality after transcatheter aortic valve implantation for severe symptomatic aortic stenosis. Am J Cardiol 2013;112:272-9.

13. D'Ascenzo F, Gonella A, Moretti C, et al. Gender differences in patients undergoing TAVI: a multicentre study. EuroIntervention 2013;9:367-72.

14. United States Life Tables 2012. Available online: https:// www.cdc.gov/nchs/data/nvsr/nvsr65/nvsr65_08.pdf

15. Blackstone EH, Suri RM, Rajeswaran J, et al. Propensitymatched comparisons of clinical outcomes after transapical or transfemoral transcatheter aortic valve replacement: a placement of aortic transcatheter valves (PARTNER)-I trial substudy. Circulation 2015;131:1989-2000.

16. Manteuffel M, Williams S, Chen W, et al. Influence of patient sex and gender on medication use, adherence, and prescribing alignment with guidelines. J Womens Health (Larchmt) 2014;23:112-9.

17. Rolnick SJ, Pawloski PA, Hedblom BD, et al. Patient characteristics associated with medication adherence. Clin Med Res 2013;11:54-65.

18. Holt E, Joyce C, Dornelles A, et al. Sex differences in barriers to antihypertensive medication adherence: findings from the cohort study of medication adherence among older adults. J Am Geriatr Soc 2013;61:558-64.

19. Smith CR, Leon MB, Mack MJ, et al. Transcatheter versus surgical aortic-valve replacement in high-risk patients. $\mathrm{N}$ Engl J Med 2011;364:2187-98.

20. Hannan EL, Samadashvili Z, Stamato NJ, et al. Utilization and 1-Year Mortality for Transcatheter Aortic Valve Replacement and Surgical Aortic Valve Replacement in New York Patients With Aortic Stenosis: 2011 to 2012. JACC Cardiovasc Interv 2016;9:578-85. 